\title{
A New System for Continuous Monitoring of Breathing and Kinetic Activity
}

\author{
Roberto Marani, Gennaro Gelao, and Anna Gina Perri \\ Electrical and Electronic Department, Polytechnic of Bari, Via E. Orabona, Bari 70125, Italy \\ Correspondence should be addressed to Anna Gina Perri, perri@poliba.it
}

Received 24 February 2010; Revised 11 June 2010; Accepted 6 July 2010

Academic Editor: Cristina E. Davis

Copyright (๑) 2010 Roberto Marani et al. This is an open access article distributed under the Creative Commons Attribution License, which permits unrestricted use, distribution, and reproduction in any medium, provided the original work is properly cited.

We present a new system for acquiring simultaneously breathing rate and kinetic activity over a period of twenty-four hours. The system is based on a couple of sensors, which are very light, absolutely noninvasive, and compatible with everyday life. The proposed breathing sensor is cheap and uses a conductive rubber as active material. An analog signal representing the breathing rate is obtained from the sensor's signal, breath by breath, without any averaging or filtering. The kinetic activity sensor is based on a tiny accelerometer whose signal is averaged and filtered, so that both sensors have voltage compatible with a slow data logging.

\section{Introduction}

In physical activity monitoring often only one parameter at a time is detected [1], since otherwise patient movement capabilities would be compromised. This is unsatisfactory since correlation among parameters is necessary to reject parameters alteration due to everyday life, but also it is necessary to detect correlated parameter alteration due to medical causes. Let us think about how everyday activities could modify heart rhythm, breathing rate, and body temperature.

Moreover the employed sensors have to be very light, noninvasive, and absolutely compatible with the ordinary day activities.

In this paper we present a new system for acquiring simultaneously some health parameters which are strongly correlated: breathing rate and kinetic activity [1].

The system is based on a couple of sensors, which are very light, absolutely non- invasive, and compatible with everyday life.

For breathing sensing we use the already known method, the plethysmography [2], involving a belt to sense the thorax dilatation, but, in order to eliminate the practical inconveniences due to the presence of metal coils on the thorax, we apply a new kind of dilatation sensor on the belt based on a conductive rubber, which is new in breathing detection, quite cheap, and sensitive.
Breathing rate observation is quite useless when no information is available on patient activity that could cause breathing rate change. Therefore we present also a kinetic activity sensor based on commercial accelerometer.

Signals from these sensors are amplified, filtered, and elaborated and fed to the ADC of a microcontroller.

Experimental results, as we will illustrate later, have shown a clear correlation between these signals. The evaluation of correlation between breathing rate and kinetic activity requires a complex mathematical procedure, which is currently under development.

Actually we can assert that the breathing and kinetic activity monitoring allows in particular to better understand the origin of tachypnea events whether they come from physical activities or not. Furthermore, in patients with lung and heart diseases, it is very useful for the doctor to understand when tachypnea begins during physical activities and how long it persist after the subject stops.

\section{Breathing Monitoring}

In past years two main methods for breathing monitoring have been developed [3-7]. In the first method, the air flow is sensed while in the second one the breast dilatation is sensed.

Air flow monitoring is accurate but is very uncomfortable, since it requires tubing or placing sensors both in 
mouth and in nose $[8,9]$. This would rule out 24-hour logging. For our project we are so forced toward the breast dilatation monitoring, which may be quite less accurate and very sensitive to arms movements but is much more comfortable.

For breast dilatation monitoring, piezoelectric strain gauge sensors are quite problematic [10], since the charge generated at typical breathing frequencies $(0.25 \mathrm{~Hz})$ can be difficultly amplified. Moreover the temperature effect and the aging on these sensors may produce a drift of the direct component of the signal. This problem could be solve by the introduction of a low-frequency filter, having a cutoff frequency less than breathing one.

Accelerometers are not suitable, because the tiny breathing acceleration available (about $0.02 \mathrm{~g}$ ) is much smaller than the gravity acceleration $g$ and body movement acceleration.

Breast dilatation monitoring is well accomplished using a breast elastic belt [11], so sensing the belt stress makes sense breathing possible.

Unfortunately some problems still remain, which are currently under development.

In fact a problem is the sensibility to the arms movements, which could trigger false breathing pulses. This is intrinsic to the belt method, but the effect is not so frequent compared to the breathing rate.

Aside from several stress sensors we have designed a new, very interesting conductive rubber sensor, which is also quite cheap, and easy tailored. Conductive rubbers are made by mixing carbon or iron powder in the chemical reactants used to produce rubbers. They have been applied as flexible conductors and as pressure sensors, but we did not find application as dilatation sensors. Indeed conductivity of these rubbers is sensitive to stress, but among the large kinds of conductive rubbers available, not all are suited for this application.

\section{The Conductive Rubber Selection}

We look for conductive rubber satisfying the following specifications:

(i) high sensitivity to the stress,

(ii) rubber should stand the stress applied to the breast belt, about $10 \mathrm{~N}$,

(iii) moderate resistivity, between $0.1 \Omega \cdot \mathrm{m}$ and $10 \Omega \cdot \mathrm{m}$.

This range of resistivity depends on measurement problems, as the sensor is supplied at constant current. In fact, for low values of resistivity, either we would have a low voltage to the sensor and therefore an amplifier would be necessary or higher currents would be required with consequent higher battery consumption not suited for an apparatus to wear for 24 hours. On the other hand, for high values of resistivity, we could have some reliability problems regarding the rubber contacts (Figure 1), which would have a higher area.

We have used a sample of conductive rubber, from Xilor, whose resistivity was only $7 \cdot 10^{-5} \Omega \cdot \mathrm{m}$, constituted by an aggregate of small conductive spheroids, about $20 \mu \mathrm{m}$ wide,

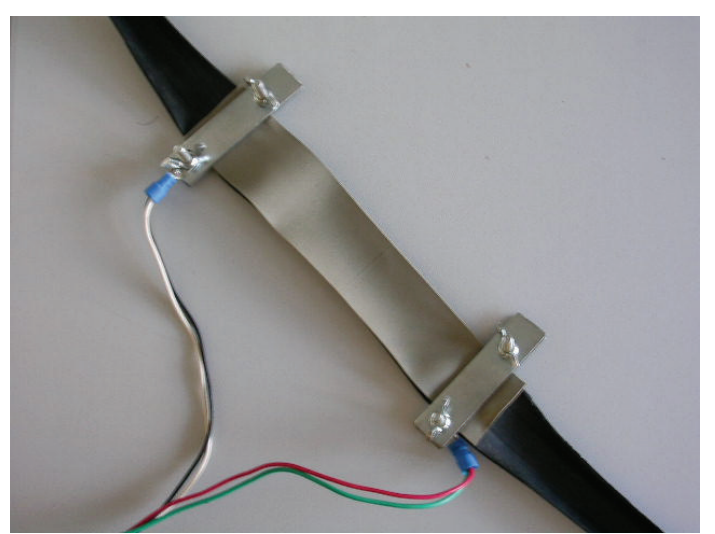

FIgURE 1: Rubber contacts.

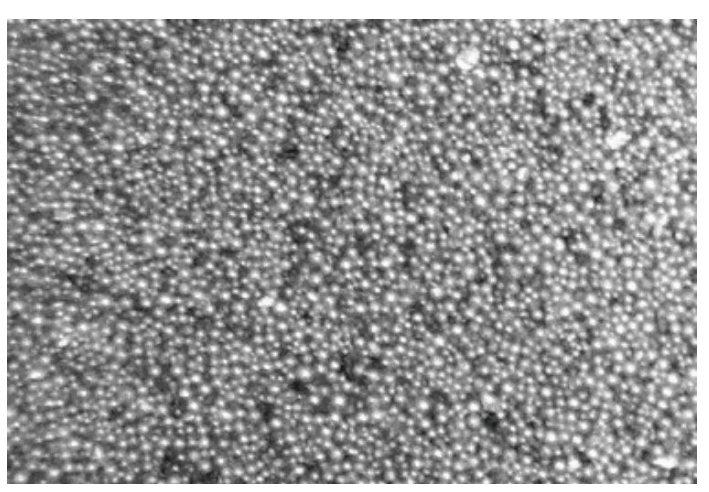

Figure 2: Microscopic view of the used conductive rubber on a broken side (visual field is $2 \mathrm{~mm} \times 1.5 \mathrm{~mm}$ ).

as shown in Figure 2, in which it is clear that the structure is composed of microspheres of rubber.

Figure 2 has been taken by an optical microscope of our electronic devices laboratory. The resistivity is controlled by the contact surface area between spheroids. This area varies according to the mechanical stress, so that resistivity is very sensitive to the mechanical stress. This material did not satisfy our initial request, because it is not so strong and has a low resistivity, but the sensibility was so high that we have selected this material to develop our prototype.

We took a sample of Xilor rubber $120 \mathrm{~mm}$ long, $20 \mathrm{~mm}$ large, and $0.3 \mathrm{~mm}$ thick that was fit in the breast belt, at the place of a piece of belt. Since the sample is not capable of standing all the belt stress, a full belt built only with this kind of conductive rubber is not feasible. To solve this problem we have added an ordinary nonconductive rubber in a mechanical parallel to this sample of Xilor rubber. We are also looking for conductive rubbers having both the sensitivity and the mechanical strength, but our actual solution with two rubbers is very satisfactory since it splits the mechanical and the electrical problem leaving us more degrees of freedom in the rubbers choice, requiring just a small amount of the more expensive conductive rubber.

Moreover, in this way we can reduce another aging problem, due to the time variation of the rubber sensibility. 

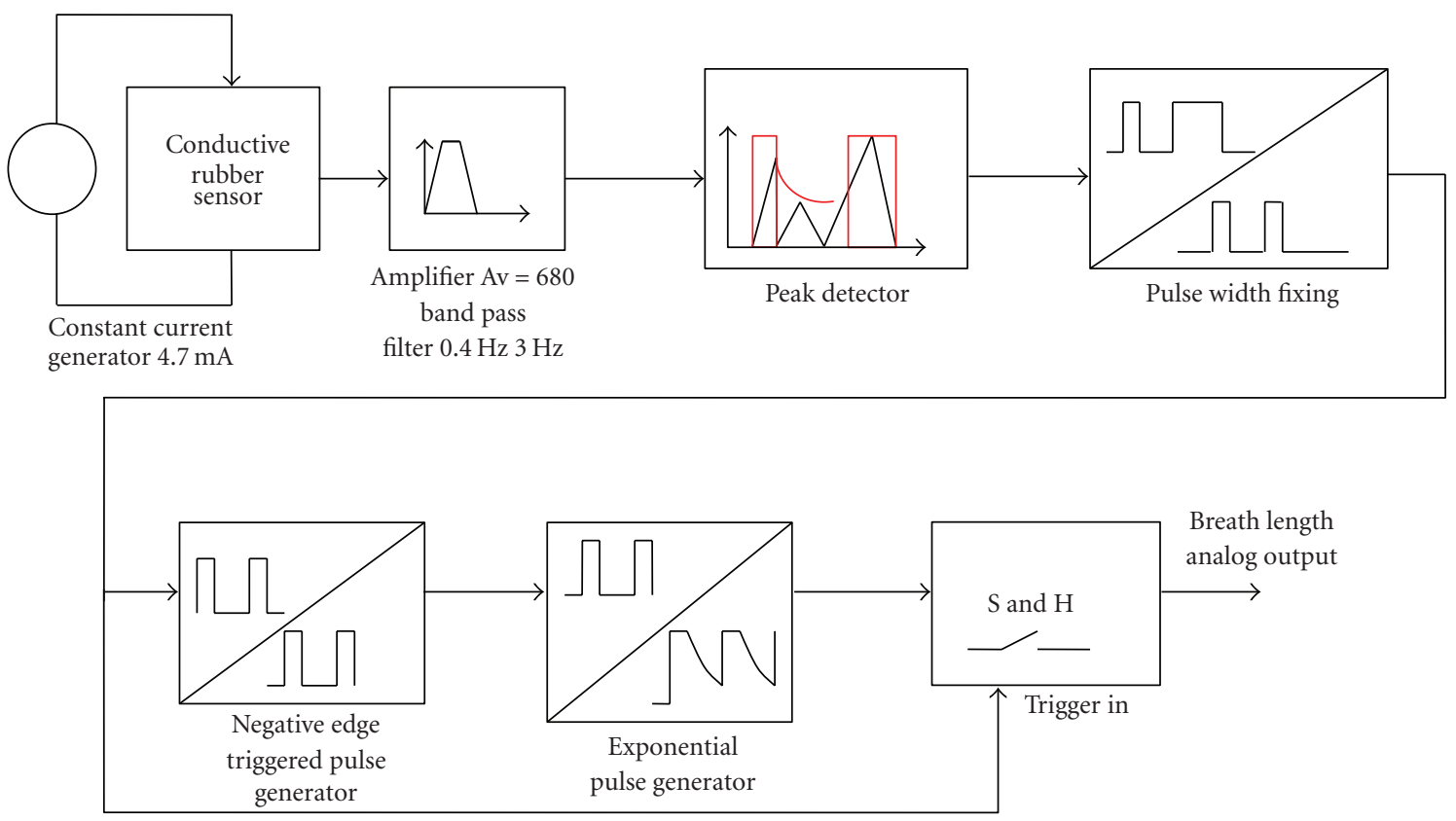

FIgURE 3: Circuit used to have a frequency-voltage conversion.

Since the sensor is not expansive, we foresee a disposable use of the sensor to overcome the rubber's aging.

Two couples of small iron plates were tightened to each end of the rubber sandwich to ensure electrical connections, as shown in Figure 1.

3.1. Electronic Interface and Experimental Results. The resistance of conductive rubber, about $1 \Omega$, was measured with the four wires method, two wires to inject a constant current and two wires to sense the voltage. We have not used the well-known Wheatstone bridge method because the sensor resistance drift, due to the aging, could require a continuous bridge balancing and, above all, because the resistance variation is not small.

Since breathing rate ranges from $0.1 \mathrm{~Hz}$ to $3 \mathrm{~Hz}$ and chest movement signal is impulsive with frequency in the frequency range $[0.4 \mathrm{~Hz}, 3 \mathrm{~Hz}]$ our front end amplifier was connected to the sensor through a first-order filter with a low cutoff frequency at $0.4 \mathrm{~Hz}$, in order to eliminate the low frequency noise of sensor, and upper cutoff frequency at $3 \mathrm{~Hz}$. Moreover we have considered the low cutoff frequency at $0.4 \mathrm{~Hz}$ because our prototype is particularly dedicated to sportsmen. Therefore we have considered the $3 \mathrm{~Hz}$ breathing rate, corresponding to 180 breaths per minute, to simulate also the transient breathlessness condition due to, for an example, a race. Furthermore it is possible to easily change the element values of the first-order filter to have a low cutoff frequency at $0.1 \mathrm{~Hz}$.

The amplified signal is sent to a peak detector (tuned for breathing rate frequencies), whose output pulses are sent to a peak shaper to have standard length pulses.

This output is already a good signal for breathing rate measurement, but, since we preferred to measure a voltage than a frequency, we fed the pulses in a frequency/voltage converter. A voltage signal for breathing rate measurement allows us to use the ADC of a microcontroller.

In order to have a breath-by-breath conversion, without contiguous period averaging, we have used the circuit shown in Figure 3, whose key elements are an exponential pulse generator and a Sample \& Hold (S\&H).

For each pulse coming from the previous circuit the exponential pulse generator is triggered, then the tail of this pulses is sampled just before the generator is retriggered. The synchronization between the $\mathrm{S} \& \mathrm{H}$ and the generator is controlled by a negative edge triggered pulse generator which sends delayed pulse to the exponential generator. Since the exponential pulse is sampled before the reset, the voltage held to the output of the S\&H is one-to-one function of the time length of the last breathing act, that is, in our case, a nonlinear map from $[0 \mathrm{~Hz}, \infty \mathrm{Hz}]$ to $[0 \mathrm{~V}, 2.5 \mathrm{~V}]$, as shown in Figure 4.

As a benefit, no average between consecutive pulses is done and the output staircase waveform is useful for a slow ADC sampling. Since this signal is available only at the end of the breath, this is unsuited for triggering warning in case of breathing stop. Whether this warning would be needed, the output of the pulse shaper (or the output of exponential generator) would be used.

Using exponential generator, the map between the frequency and the voltage is nonlinear. This is not so bad, since nonlinearity could be corrected on the remote computer display and the exponential allows to map any time length to a finite voltage. Furthermore, since the output voltage is fed to an ADC, and because of the nonlinearity, the best resolution would be given at the most common breathing rate while the uncommon rates would have lower resolution. 


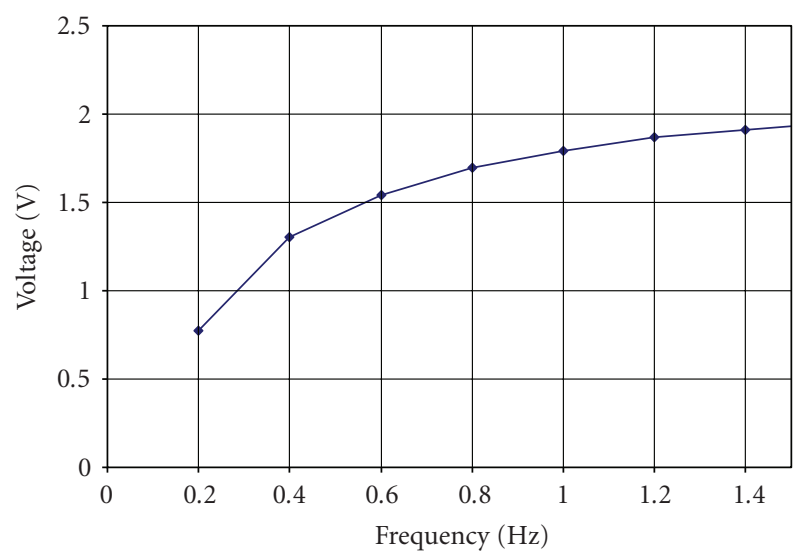

Figure 4: Frequency-voltage conversion diagram.

Of course, noise and time delay would cut off the far ends of the interval $[0 \mathrm{~Hz}, \infty \mathrm{Hz}]$ from the map, but the remaining interval is still wide: the system has been tested on the wide interval $[0.05 \mathrm{~Hz}, 6 \mathrm{~Hz}]$.

Figures 5 and 6 show the signal obtained with the described electronic setup.

As shown in Figure 5, the signal is clear and noiseless; the figure also shows the signal from the peak detector which is well behaved.

The system has been successfully tested on a wide breathing rates interval (see Figure 6), but still the aging problem remains.

In order to allow a test on a wider frequency range, Figure 6 has been obtained using an electronic pulse generator whose signal was fed to the frequency voltage converter.

Indeed the rubber resistivity raised tenfold after few hours of usage, when the rubber was fit in the belt with the dilatation method. While this could be compensated with an automatic gain control at the front-end amplifier, it would be better to measure compression effects on rubber conductivity.

\section{Physical Activity Sensor Monitoring}

The physical activity is important also to understand the medical meaning of heart and breath dynamics. For example, let us think how the meaning of a high heart rate could be different whether the subject is running or standing.

Physical activity monitoring is also very useful to understand objectively the lifestyle of a patient to evaluate his daily energetic expenses.

4.1. Sensor. We have observed that root mean square (r.m.s.) values of acceleration (passed through a high-pass filter) on the body surface are fully correlated with walking speed. Experimental data collected in previous experiences with other accelerometer show that vertical acceleration on the shoulder of a running patient peaks from $-1 \mathrm{~g}$ to $2 \mathrm{~g}$, while power spectrum spans up to $20 \mathrm{~Hz}$ (Figure 7). Peaks come from each impact of feet on ground.

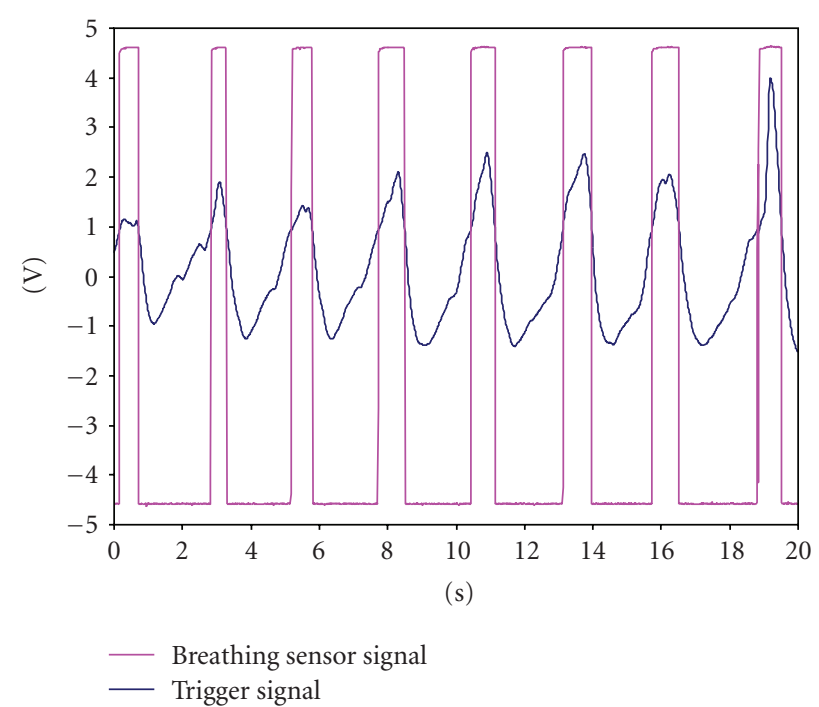

FIGURE 5: Breathing act identification: the square pulses are from peak detector while the wavy signal is the amplified sensor signal.

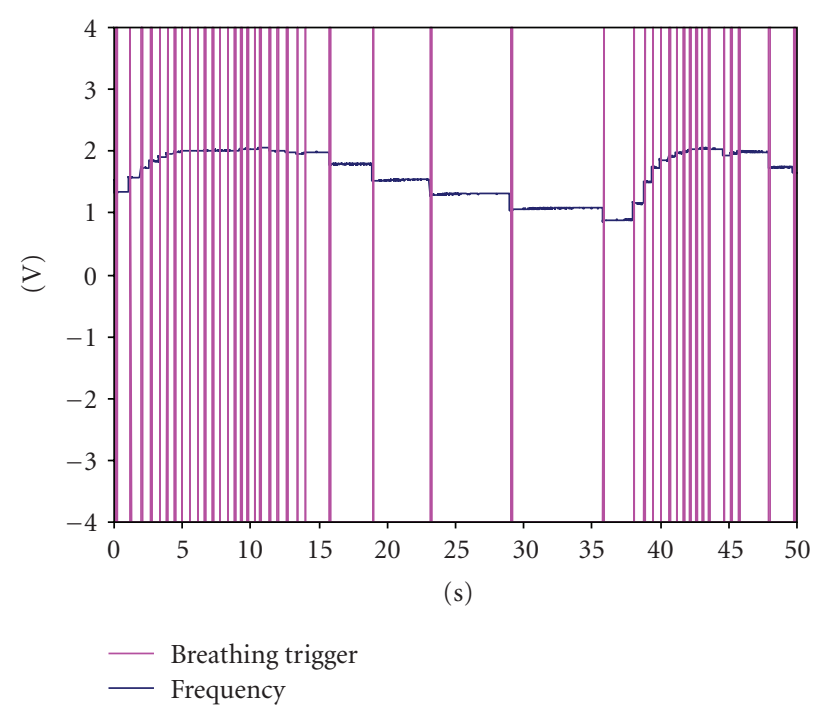

FIGURE 6: Frequency measurement: the vertical pulses are trigger pulses from the pulse width fixing block while the wavy line is the voltage converted frequency at the circuit output.

This signal includes a contribute coming from the gravity, that is, $g(\cos \theta)$ where $\theta$ is the angle between the sense direction of the accelerometer and the gravity. The variable $\theta$ is not constant at all when the accelerometer is fixed on the clothes of a patient but varies widely when a subject bows or stands up. Fortunately the power spectrum of $\theta$ is concentrated at frequency below $1 \mathrm{~Hz}$, much lower than the frequencies of the acceleration of a walking or running patient. In conclusion it is necessary and also enough to use a high-pass filter to cut off the gravity.

We have chosen the accelerometer ADXL103 (by Analog Devices) whose characteristics are ideal for our application. While the band is much larger than what we need (but this is not a problem), the noise density is low, $10^{-4} \mathrm{~g} / \mathrm{Hz}^{1 / 2}$, and 
useful for good measurements. Its power dissipation is low, it takes $0.7 \mathrm{~mA}$ at $5 \mathrm{~V}$, and very useful since our system is battery powered. It is also quite cheap (about 9 ).

A particular explanation should be deserved to our interest in the sensitivity range: $\pm 1.7 \mathrm{~g}$. This is a bit lower than maximum measurement on a running patient, so this would cause accelerometer signal clipping and would create a progressive saturation of our circuit output signal. We do not foresee a complete saturation, since this would happen only if the patient runs in a very heavy way.

This is not a real limitation, since a running patient could be statistically marginal and signal partial saturation would be marginal; furthermore for athletic application a new device would be used with larger sensitivity range and different tuning. On the other hand, the cut at $\pm 1.7 \mathrm{~g}$ cuts off the high acceleration peaks, up to several tens of g, coming from collisions of accelerometer with the environment. In absence of clipping, high g peaks could ruin the 24-hour averages of r.m.s. acceleration.

4.2. Electronic Interface and Experimental Results. The signal chain is quite simple and requires a band pass filter to cut off low frequencies at $0.7 \mathrm{~Hz}$ (related to gravity) and high frequencies at $20 \mathrm{~Hz}$ to clean unforeseen unwanted signal outside the signal band. The upper part of Figure 8 shows the output signal.

The filtered signal is passed to a cheap and effective r.m.s. converter, the AD737, whose precision is enough for our purposes. The r.m.s. converter output is filtered to cut off frequencies over $0.1 \mathrm{~Hz}$ to kill off the residual ripple observed on running patients. The lower part of Figure 8 shows this signal.

The signal is clean, and spanned voltage range is matched to input span of the ADC of our wearable unit.

\section{Conclusion}

In this paper we have presented a new system for acquiring simultaneously the breathing rate and the kinetic activity.

The system is based on a couple of sensors, which are very light, absolutely noninvasive, and compatible with every day life.

For breathing sensing we have used an already known method involving a belt to sense the thorax dilatation, but, in order to eliminate the practical inconveniences due to the presence of metal coils on the thorax, we have applied a new kind of belt dilatation sensor on the belt.

In order to have information on patient activity, we have proposed also a kinetic activity sensor based on commercial accelerometer.

Signals from these sensors are amplified, filtered, and elaborated and fed to the ADC of a micro-controller.

Experimental results have shown a clear correlation between breathing rate and kinetic activity, which actually is not possible to quantify, but we are beginning to investigate.

The proposed kinetic sensor, based on commercial accelerometer, has been tested on several subjects, for each patient it was clearly possible to recognize whether the subject was standing, walking, or running.

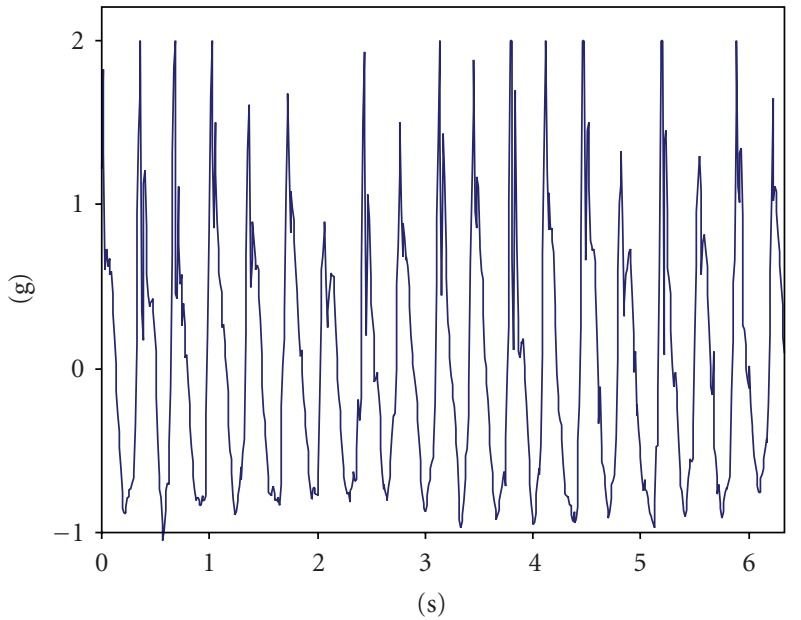

FIgure 7: Acceleration signal detected on a running man. The acceleration is measured in $\mathrm{g}=9.81 \mathrm{~m} / \mathrm{s}^{2}$.

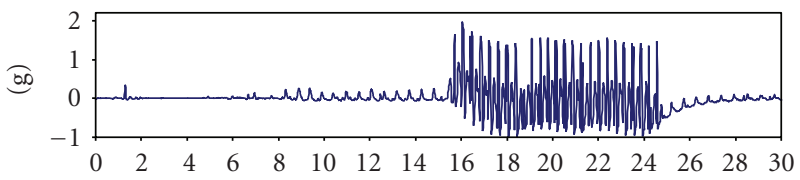

(s)

(a)

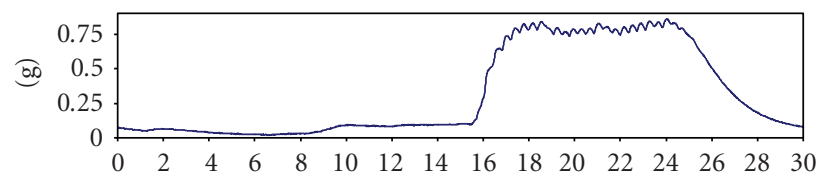

(s)

(b)

Figure 8: (a): Accelerometer signal. (b): r.m.s. filtered signal.

The observed signal is not only correlated to the physical activity but also to the weight of the subject and (we suppose) also the way the subject walks. For simple qualitative analysis this is not a problem, but, if a quantitative analysis is required, a personal calibration would be performed, or simply a statistical parameterization of calibration on some biological parameter, for example, weight, height, sex, and age.

With this calibration, we hope that this physical activity measurement would allow a good quantitative estimator of the energetic expenses to what concerns walking and running, and we hope that, using also other biological parameters to evaluate basic metabolism, it could be possible to estimate the daily energetic expenses.

This would be very interesting since the available methods that measure the $\mathrm{CO}_{2}$ (with mouth and nose tubing [8]) and the heat production (in a calorimetric box) are not suited for 24-hour measurements. 


\section{References}

[1] W. Welkowitz and S. Deutsch, Biomedical Instruments: Theory and Design, Academic Press, New York, NY, USA, 1976.

[2] K. Konno and J. Mead, "Measurement of the separate volume changes of rib cage and abdomen during breathing," Journal of Applied Physiology, vol. 22, no. 3, pp. 407-422, 1967.

[3] J. G. Webster, Ed., Medical Instrumentation, John Wiley \& Sons, New York, NY, USA, 1998.

[4] J. Moore and G. Zouridakis, Eds., Biomedical Technology and Devices Handbook, CRC Press, Boca Raton, Fla, USA, 2004.

[5] F. P. Branca, Fondamenti di Ingegneria Clinica, Springer, Berlin, Germany, 2000.

[6] T. Togawa, T. Tamura, and P. A. Oberg, Biomedical Transducers and Instruments, CRC Press, Boca Raton, Fla, USA, 1997.

[7] L. Tarassenko, L. Mason, and N. Townsend, "Multi-sensor fusion for robust computation of breathing rate," Electronics Letters, vol. 38, no. 22, pp. 1314-1316, 2002.

[8] A. Lay-Ekuakille, G. Vendramin, and A. Trotta, "Spirometric measurement postprocessing: expiration data recovery," IEEE Sensors Journal, vol. 10, no. 1, pp. 25-33, 2010.

[9] C. Wei, C. Lin, and I. Tseng, "A novel MEMS respiratory flow sensor," IEEE Sensors Journal, vol. 10, no. 1, pp. 16-18, 2010.

[10] P. Bonato, "Wearable sensors/systems and their impact on biomedical engineering," IEEE Engineering in Medicine and Biology Magazine, vol. 22, no. 3, pp. 18-20, 2003.

[11] K.-A. Kim, I.-K. Lee, S.-S. Choi, T.-S. Lee, and E.-J. Cha, "Conductive rubber belt to monitor respiratory changes," in Proceedings of the 5th IEEE Conference on Sensors, pp. 74-76, Daegu, Korea, October 2006. 

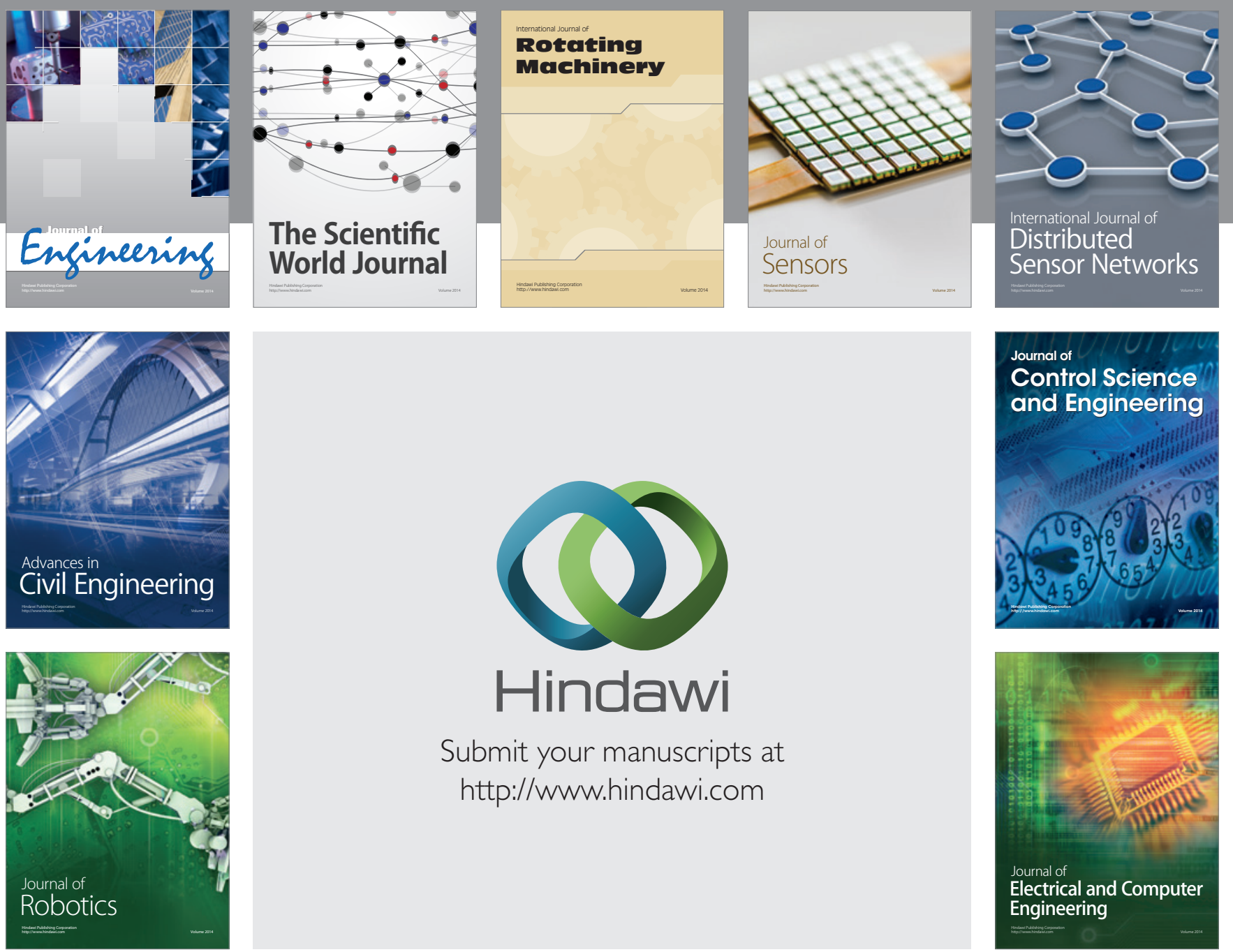

Submit your manuscripts at

http://www.hindawi.com
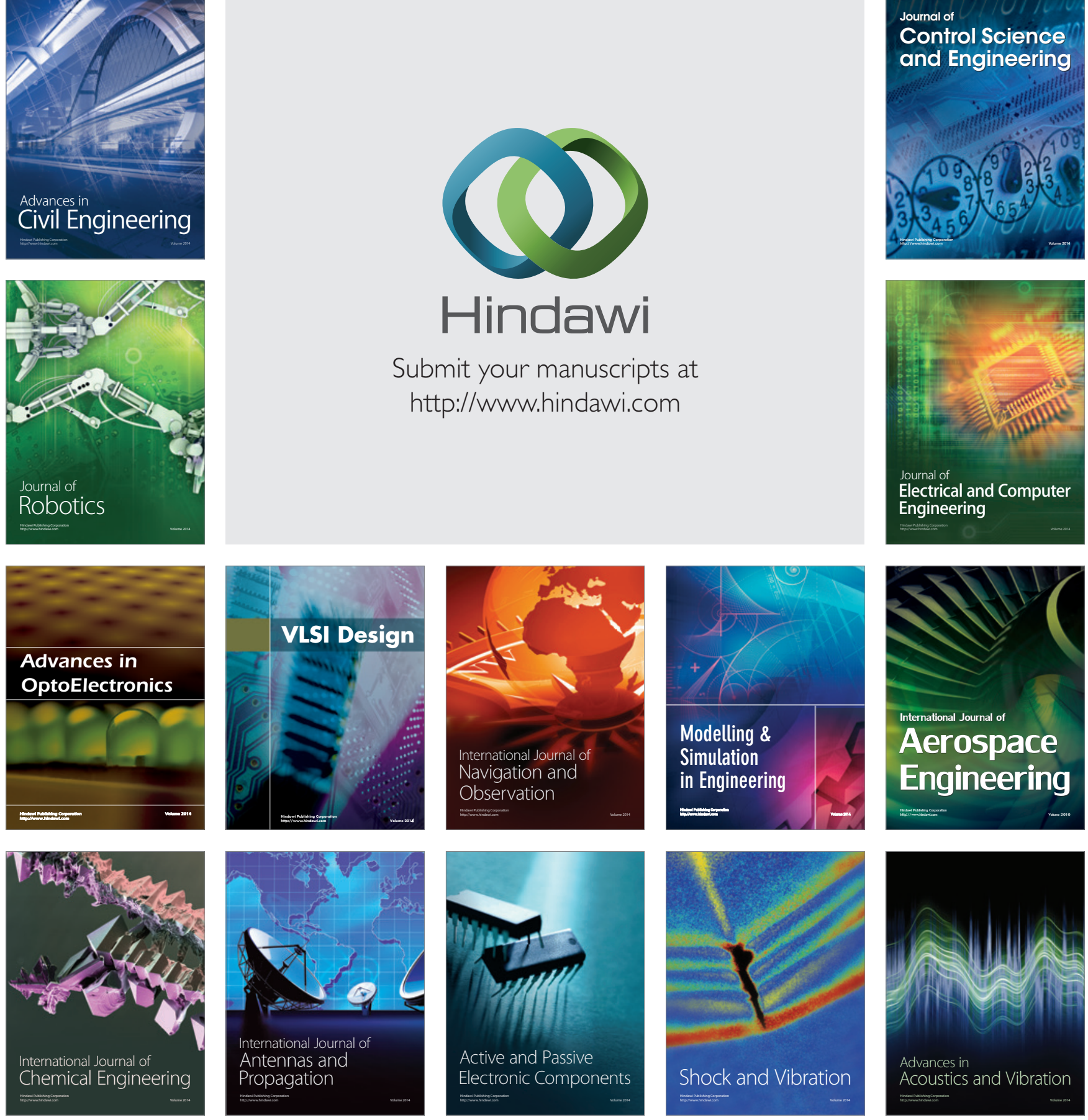